\title{
Bemerkung über die Schönfeld'schen Beobachtungen des vollen Lichtes bei Algol.
} Von Dr. F. Scheiner.

In Nr. 3072 der Astr. Nachr. berichtet Herr Plassmann in seiner Notiz Ueber die wahre Gestalt der Algol-Curve über seine Beobachtungen des vollen Lichtes von Algol, nach denen zwei deutliche Minima bei $2 \mathrm{I}^{\mathrm{h}}$ und $47^{\mathrm{h}}$, sowie drei Maxima hervortreten sollen. Bei dieser Gelegenheit erwähnt Herr Plassmann, dass die von mir bearbeiteten Schönfeld'schen Beobachtungen - Untersuchungen über den Lichtwechsel Algols nach den Mannheimer Beobachtungen von Professor Schönfeld in den Jahren 1869 bis 1875 , Bonn I882 - dieselben Maxima und Minima ergeben, sofern man sie in geeigneter Weise zusammenzieht. Ich muss dieser Behauptung entschieden widersprechen und zwar aus denselben Gründen, welche mich damals veranlasst haben, die geringen Schwankungen in der Helligkeit des vollen Lichtes, welche die Schönfeld'schen Beobachtungen zeigen, als zufällige zu betrachten, wie dies Schönfeld bereits vorher für seine früheren Beobachtungen gethan hat. Der wahrscheinliche Fehler eines aus ungefähr ro Beobachtungen gebildeten $N$ für das volle licht betrug \pm 0.062 Stufen, während der entsprechende aus der eigentlichen Lichtcurve sich zu \pm 0.155 ergab, also um mehr als das Doppelte

Potsdam, Kgl, Observatorium, r892 Juni. grösser war. Es handelte sich mithin darum, die auffallende Kleinheit des ersten wahrscheinlichen Fehlers zu erklären, und nicht darum, denselben durch Einführung von Einbiegungen in die gerade Iinie, durch welche die Beobachtungen des vollen Lichtes dargestellt wurden, noch weiter zu verkleinern. Diesen Umstand scheint Herr Plassmann gänzlich übersehen zu haben. Ich habe damals in voller Uebereinstimmung mit Schönfeld als Erklärung für die Kleinheit des wahrscheinlichen Fehlers folgendes angefürt: »Ein Theil dieser Verkleinerung muss also wohl einer Abhängigkeit zugeschrieben werden, welche auch die Beobachtungen verschiedener Abende etwas beeinflusst hat, ein Umstand, der gerade bei diesen Beobachtungen trotz grösster Sorgfalt und Unbefangenheit nicht zu vermeiden sein wird «.

Ein Urtheil über die Realität der Resultate und Hypothesen, welche Herr Plassmann aus seinen Beobachtungen gezogen hat, unterlasse ich; jedenfalls aber glaube ich, durch den oben gegebenen Hinweis, dass die Beobachtungen Schönfeld's keine reellen Schwankungen des vollen Lichtes Algols erkennen lassen, im Sinne des Verstorbenen gehandelt zu haben.

\section{Ephemeride des Planeten 1892 C (Wolf).}

Aus den Heidelberger photographischen Beobachtungen

\begin{tabular}{|c|c|c|c|}
\hline 1892 & M. Z. Gr. & $\alpha$ app. & $\delta$ app. \\
\hline Sept. I & $1 x^{\mathrm{h}} 28^{\mathrm{m}} \cdot 5$ & $23^{\mathrm{h}} 55^{\mathrm{m}} 27^{\mathrm{s}} .68$ & $-4^{\circ} 10^{\prime} 41 " 5$ \\
\hline I 3 & 22.5 & 2346 & $34 \cdot 5$ \\
\hline
\end{tabular}

habe ich die folgende Kreisbahnephemeride für I $^{\mathrm{h}}$ M. $Z$. Berlin berechnet.

Berlin, Kgl. Recheninstitut, 1892 Sept. 16.
F. Scheiner.

\begin{tabular}{|c|c|c|c|c|c|c|c|}
\hline \multicolumn{2}{|c|}{1892} & \multicolumn{3}{|c|}{$\alpha$} & \multicolumn{2}{|c|}{$\delta$} & $\log A$ \\
\hline \multicolumn{2}{|c|}{ Sept. 20} & $23^{h}$ & $40^{1 n}$ & $4^{5}$ & $-6^{\circ}$ & $47: 6$ & 0.1513 \\
\hline & 24 & & $3^{6}$ & 46 & 7 & 18.4 & \\
\hline & 28 & & 33 & 35 & 7 & $47 \cdot 3$ & o. I 576 \\
\hline Oct. & 2 & & 30 & 37 & 8 & I 3.6 & \\
\hline & 6 & & 27 & 55 & 8 & 37.1 & o. 1687 \\
\hline & IO & & 25 & 32 & 8 & $57 \cdot 5$ & \\
\hline & I 4 & 23 & 23 & 31 & -9 & 14.4 & 0.1837 \\
\hline
\end{tabular}

A. Berberich.

\section{Beobachtung des Cometen 1892 ... (Brooks Aug. 27).}

1892 Sept. 16 I $2^{\mathrm{h}} 57^{\mathrm{m}} 5^{2^{\mathrm{s}}}$ M. Z. Kopenh. $\Delta \alpha=+0^{\mathrm{m}} 29^{\mathrm{s}} \cdot 39 \quad \Delta \delta=+3^{\prime} 5^{\prime \prime} \cdot 8$ Vgl. $20.4 \quad \alpha$ app. $=6^{\mathrm{h}} 47^{\mathrm{m}} 17^{\mathrm{s}} .09$ (9.584n) d app. $=+29^{\circ} 42^{\prime} 25^{\prime \prime} 9(0.804) \quad$ red. ad l. app. $=+\mathrm{r}^{\mathrm{s}} .52+3^{\prime \prime 6}$.

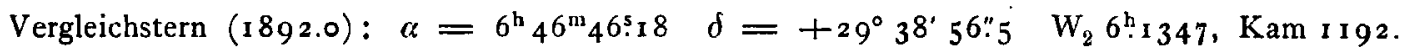

Kopenhagen 1892 Sept. I 7 .

C. F. Pechüle.

Berichtigungen $2 u$ Nr. 3062 Bd. 128 S. 254 Z. I 7 r. o. stalt: 一ís lies: +16s

$\gg, 3100 \gg 130 \mathrm{~S} .52$ \%. I 3 v. u. die Gleichung für $W$ lies:

$$
W=\psi+H\left(g+i \log \operatorname{tg} \frac{s}{2}\right)=H\left(\gamma+i \log \operatorname{tg} \frac{\sigma}{2}\right) \text {. }
$$

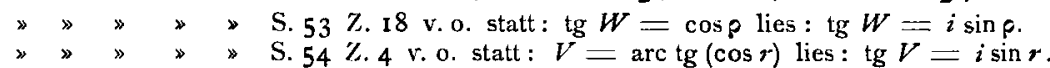

$\gg \gg \gg \gg$, S. 54 Z.6 v. o. statt: $\rho=\arccos (\operatorname{tg} W) \operatorname{lies}: i \sin \rho=\operatorname{tg} W$.

Inhalt zu Nr. 3117. T. Wand. Ueber die Integration der Differentialgleichungen, welche die Bewegungen eines Systems von Punkten bestimmen. 377. - O. Knopf. Comet 1892 . . (Brooks Aug. 27). 389. - F. Scheiner. Bemerkung iiber die Schönfeld'schen Beobachtungen des vollen Lichtes bei Algol. 391. - A. Berberich. Ephemeride des Planeten 1892 C (Wolf). 391. - C. F. Pechüle. Beobachtung des Cometen 1892... (Brooks Aug. 27). 391. - Berichtigungen. 391. 